\section{Musculoskeletal ultrasound: athletic injuries of the lower extremity}

\author{
Nathaniel B. Meyer, Jon A. Jacobson, Vivek Kalia, Sung Moon Kim \\ Department of Radiology, University of Michigan Hospital, Ann Arbor, MI, USA
}

Athletic injuries of the lower extremities are commonly encountered in clinical practice. While some pathology can be diagnosed on physical exam, others are a clinical dilemma with nonspecific symptomatology. In these situations, ultrasound imaging can be utilized as an exceptional diagnostic tool, offering unique advantages over other imaging modalities. This article will review the imaging characteristics of commonly encountered athletic injuries of the lower extremity.

Keywords: Ultrasonography; Athletic injuries; Musculoskeletal diseases; Tendonopathy; Dynamic

\section{Introduction}

Ultrasound is emerging as the preferred imaging modality to evaluate a range of lower extremity athletic injuries. In addition to high spatial resolution, an ultrasound evaluation provides several advantages allowing one to perform a dynamic evaluation tailored to the patient's chief complaint. In this setting, the patient can provide real-time feedback, confirming symptom replication at suspected sites of abnormality. Additional information is obtained from color Doppler imaging, as well as by using graded transducer pressure to assess pain, lesion compressibility, and fluid content. Anatomic structural integrity can be tested during stress maneuvers, and pathologies present only with certain positioning or with movement can be documented. Furthermore, advances in technology are making an ultrasound evaluation increasingly less expensive and portable providing diagnostic capabilities in the clinic or even on the sidelines of sporting events [1]. However, unlike other cross-sectional imaging modalities, ultrasound is relatively operator dependent. Thus, it is extremely important that one is confident in their ability to identify frequently encountered pathology and that they have a working knowledge of relevant anatomy. This article reviews the imaging of common sports injuries involving the lower extremities.

Hip

\section{Snapping Hip Syndrome}

Snapping hip syndrome can refer to several distinct entities in which the patient feels an abnormal snapping sensation, and in some cases pain, during hip movement. Broadly, these can be divided into intra-articular and extra-articular types, where intra-articular types are the result of intra-articular bodies or prior trauma to the joint and are not well evaluated on ultrasound [2]. Extra-articular causes are well evaluated on dynamic ultrasound and are further sub-typed as external or internal by

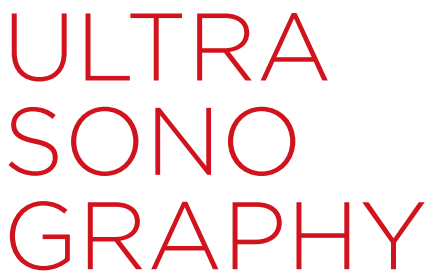

REVIEW ARTICLE

https://doi.org/10.14366/usg.18013 pISSN: 2288-5919 elSSN: 2288-5943 Ultrasonography 2018;37:175-189

Received: March 20, 2018

Accepted: April 14, 2018

Correspondence to: Nathaniel B. Meyer, MD, Department of Radiology, University of Michigan, 1500 East Medical Center Drive Ann Arbor, MI 48109, USA

Tel. +1-734-936-4365

Fax. +1-734-936-9723

E-mail: nbmeyer@med.umich.edu

This is an Open Access article distributed under the terms of the Creative Commons Attribution NonCommercial License (http://creativecommons.org/ licenses/by-nc/3.0/) which permits unrestricted noncommercial use, distribution, and reproduction in any medium, provided the original work is properly cited.

Copyright (C) 2018 Korean Society of Ultrasound in Medicine (KSUM)

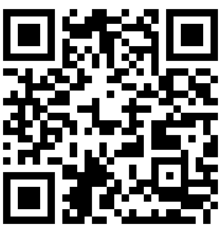

How to cite this article:

Meyer NB, Jacobson JA, Kalia V, Kim SM. Musculoskeletal ultrasound: athletic injuries of the lower extremity. Ultrasonography. 2018 Jul;37(3):175-189. 
location $[2,3]$.

Internal hip snapping, visualized at the level of the anterior inferior iliac spine with the transducer in the axial oblique plane, is the result of abnormal movement of the iliopsoas complex. At this level, the psoas major tendon is present with its muscle fibers located medially and the iliacus muscle fibers located anterolaterally (Fig. 1). As the patient flexes, abducts, and externally rotates the hip, the psoas major tendon rotates anterolaterally around the medial iliacus muscle fibers. Normally, the psoas major smoothly rotates back into position as the patient straightens the leg; however, in the snapping hip, the medial fibers of the iliacus become temporarily entrapped between the psoas major tendon and the superior pubic ramus (Fig. 1). In the later phase of leg straightening, the interposed iliacus muscle fibers suddenly move laterally and the psoas major tendon snaps against the superior pubic ramus [2-4]. Other biomechanical etiologies for symptoms at this location include a bifid psoas tendon or movement of the tendon over a paralabral cyst [2-5].

External hip snapping occurs at the greater trochanter and is the result of abnormal movement of the iliotibial band or anterior gluteus maximus over the greater trochanter. Normally, these two structures glide smoothly over the lateral facet as the patient flexes the hip, but either may be abnormally restrained during the early phase of flexion, until it is suddenly released, springing anteriorly, and resulting in a snapping sensation (Fig. 2). Ultrasound may demonstrate a thickened iliotibial tract or band with surrounding edema $[2,3]$.

\section{Athletic Pubalgia}

Athletes with pubic symphyseal and groin pain, termed athletic pubalgia, often play sports involving sprinting or rapid changes in direction, including soccer, football, and hockey [6]. There are multiple etiologies; however, the term "sports hernia" is a misnomer, since symptoms are often not related to a true hernia [7]. Instead, the most frequent pathology involves the common aponeurosis, which extends contiguously from each inferior rectus abdominis, over the pubis, and to the ipsilateral adductor longus [6-9].

Imaging features of athletic pubalgia include a thickened and hypoechoic common aponeurosis with or without anechoic clefts, and irregularity of the underlying pubis cortex (Fig. 3). Partial or complete tears at the adductor or rectus musculature are seen as anechoic clefts or retraction of the muscle fibers with interposed heterogeneous hematoma, respectively (Figs. 3, 4). Fluid distention of the symphysis pubis capsule may represent a symphyseal injury or osteoarthritis. These entities should also be considered in the work up of athletic pubalgia [7].

\section{Rectus Femoris Injury}

Abnormalities in the proximal rectus femoris are prevalent in runners and soccer players $[10,11]$. The rectus femoris arises as two

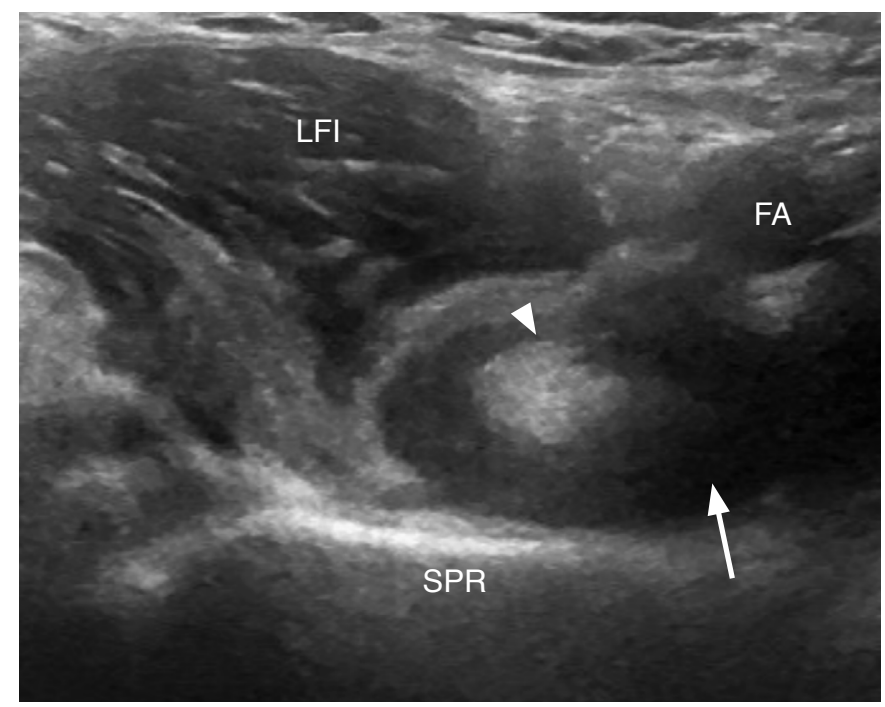

A

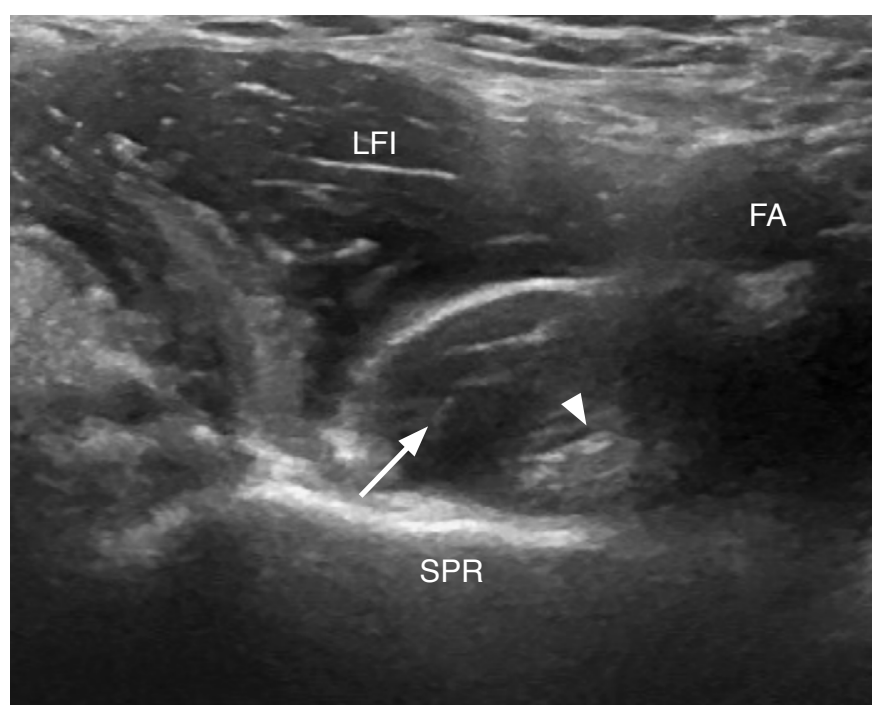

B

Fig. 1. A 17-year-old girl with right snapping iliopsoas.

A. Ultrasonography in oblique transverse plane (right side of image is medial) with patient hip in frog-leg position shows medial fibers of the iliacus (arrow) temporarily trapped between the psoas major tendon (arrowhead) and superior pubic ramus (SPR). B. With leg extension, medial fibers of the iliacus move laterally (arrow), and the psoas major tendon (arrowhead) snaps down against the superior pubic ramus, returning to normal resting anatomical position. LFI, lateral fibers iliacus; FA, femoral artery. 
tendons. The direct head, also known as the straight or anterior head, originates from the anterior inferior iliac spine, while the indirect head, also known as the reflected or posterior head, is located slightly laterally and posteriorly, arising along the lateral acetabular rim [12]. Both heads need to be imaged for a complete examination. To find the indirect head, begin with your transducer short axis over the direct head at the anterior inferior iliac spine, then move the transducer laterally over the acetabulum and rotate the lateral aspect of the transducer 30 degrees inferiorly to visualize the indirect head in long axis [12].

Imaging findings of proximal rectus tendon injury is similar to other tendon pathology. Hypoechoic and possibly enlarged tendon fibers representing tendinosis, anechoic clefts indicating partial tearing, and complete disruption with incongruous fibers and heterogeneous hypoechoic intervening hematoma can all be identified [11]. Calcium hydroxyapatite deposition (calcific tendinosis) may also be identified as irregular areas of hyperechogenicity with posterior acoustic shadowing [13].

Although the above-mentioned tendon pathologies are more familiar, the distinctive musculotendinous junction anatomy of the rectus femoris leads to a unique pattern of injury. The musculotendinous junction of the indirect head extends from near

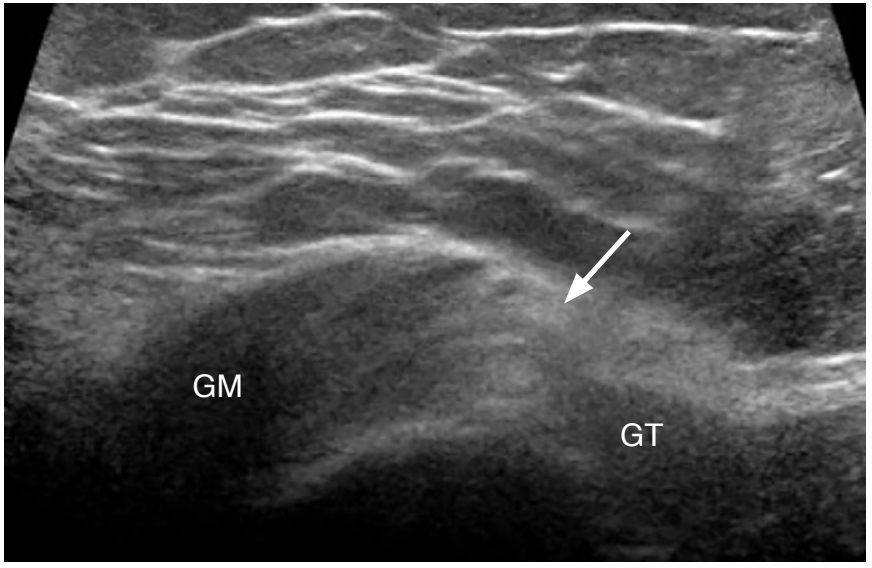

A

Fig. 2. A 20-year-old woman with right snapping iliotibial tract.

A. Transverse ultrasonography of the lateral hip (left side of image is posterior) over greater trochanter (GT) demonstrates a thickened iliotibial tract or band (arrow). B. With hip flexion, the iliotibial tract (arrow) moves anteriorly and snaps over the greater trochanter. GM, gluteus maximus.

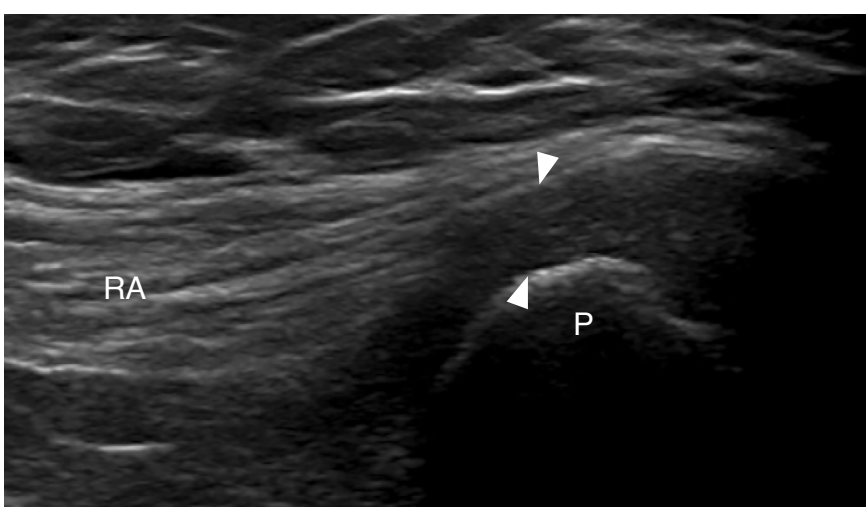

A

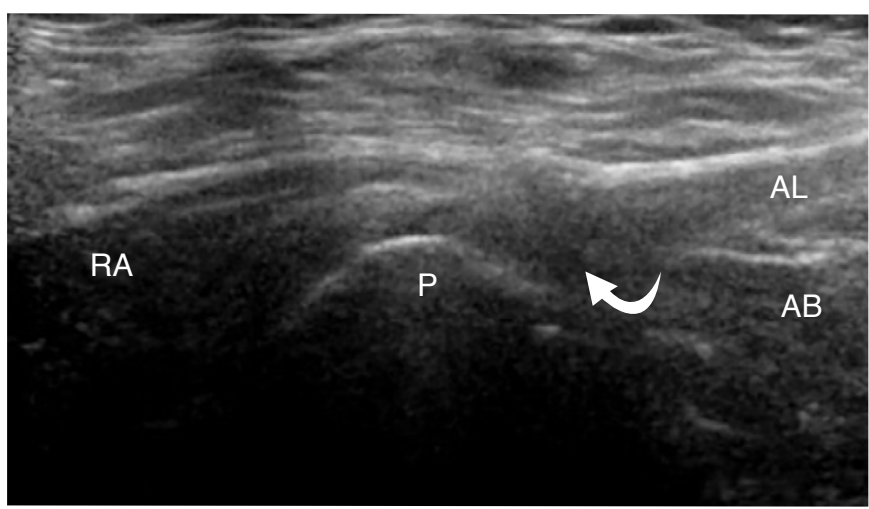

B

Fig. 3. Athletic pubalgia.

A. Ultrasonography of the anterior lower abdominal wall long axis to the rectus abdominus (RA) in a 24-year-old man demonstrates a thickened, hypoechoic common aponeurosis (arrowheads) and irregularity of the underlying pubic (P) cortex. B. Similar findings are seen in a 34-year-old woman but with additional small hypoechoic clefts (curved arrow), representing partial-thickness tears at the origin of the adductor longus (AL). AB, adductor brevis. 
the hip to approximately two-thirds of the distance to the knee with the tendon located centrally. Injury of this central aponeurosis can result in edema or hematoma surrounding the tendon, resulting in a "bullseye" appearance (Fig. 5) $[10,14,15]$. In the young adult, anterior inferior iliac spine physeal avulsion injury should be recognized if there is focal edema, widening, or irregularity at the physis [11].

\section{Knee}

\section{Quadriceps Tendon Injury}

Athletic injury to the quadriceps tendon is characteristically found in basketball or volleyball players, secondary to repeated jumping, but can also be the result of forceful flexion occurring with rapid deceleration while running $[16,17]$. Because the rectus femoris spans two joints, it is the most frequently injured component of the quadriceps femoris [18]. At the distal thigh, the quadriceps tendon is comprised of three layers. The superficial layer arises from

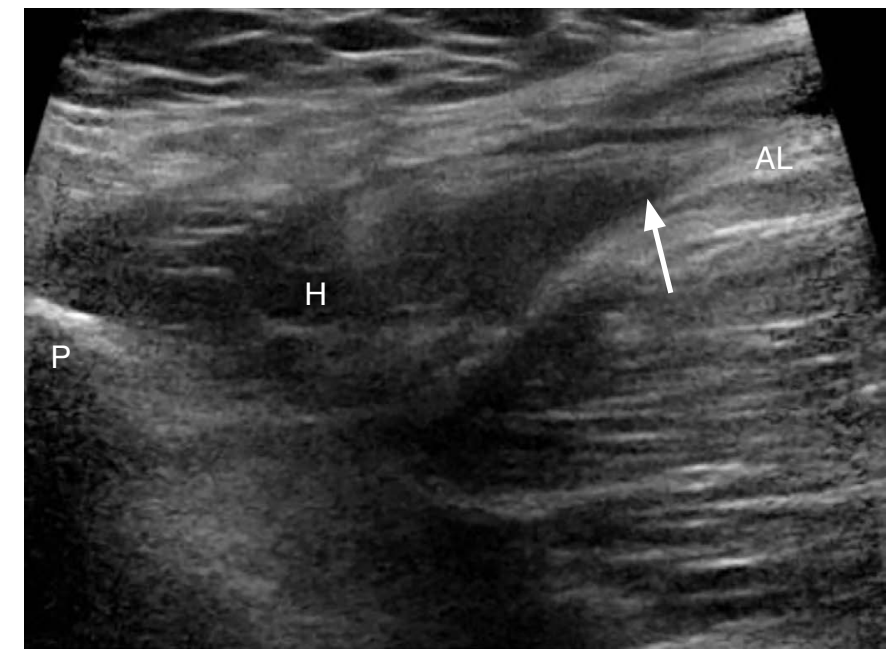

Fig. 4. A 36-year-old man with left adductor longus full-thickness tear. Ultrasonography of long axis to the adductor musculature demonstrates a full-thickness tear of the adductor longus tendon (AL) with heterogeneous, hypoechoic fluid representing hematoma $(\mathrm{H})$ interposed between the retracted tendon (arrow) and pubic bone (P).

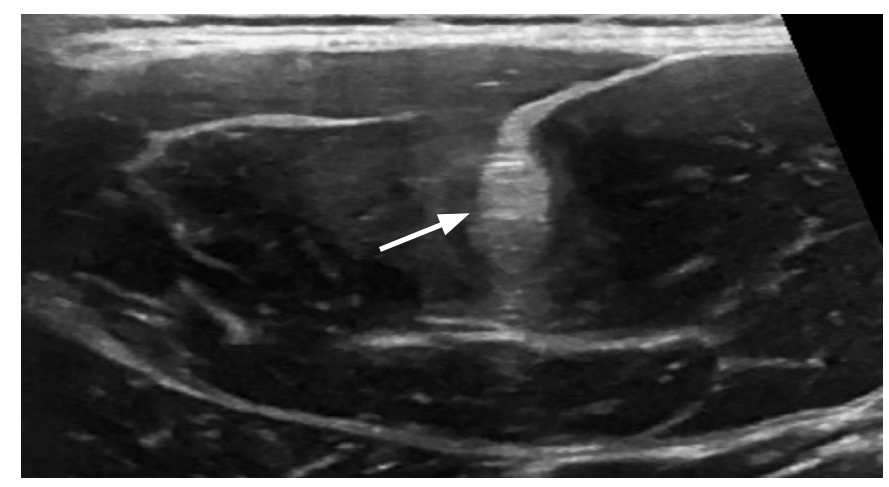

A

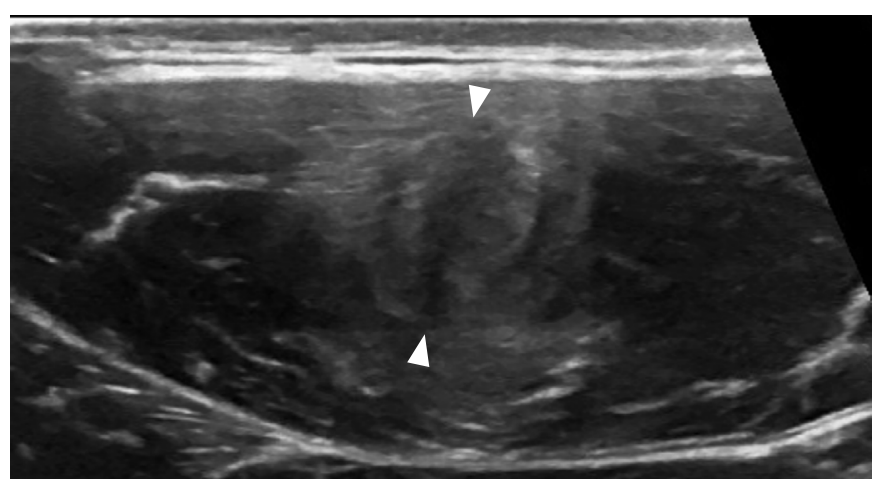

B

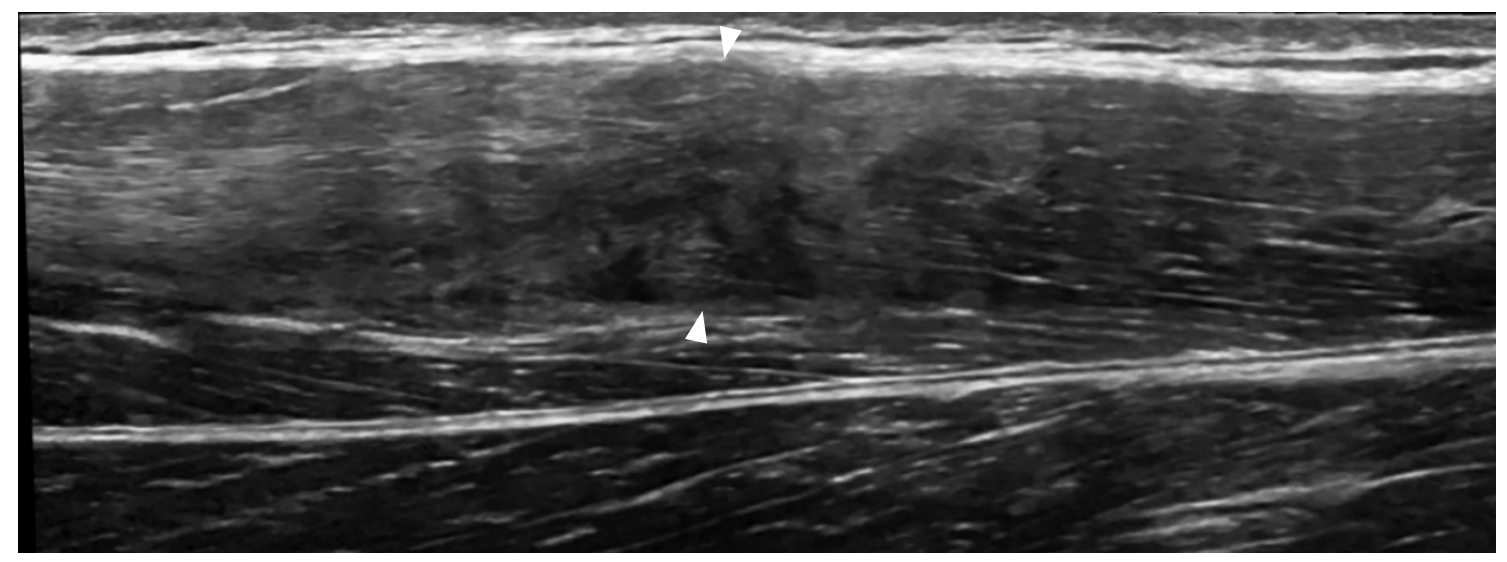

C

Fig. 5. A 13-year-old boy with indirect head rectus femoris tear.

A. Proximal transverse ultrasonography demonstrates a slightly thickened, but intact rectus femoris indirect head tendon (arrow). B. Distally, the tendon is thickened, irregular, and hypoechoic (arrowheads) with the characteristic "bullseye" appearance. C. Long-axis ultrasonography demonstrates discontinuous fibers and hematoma (arrowheads) at the level of $B$. 


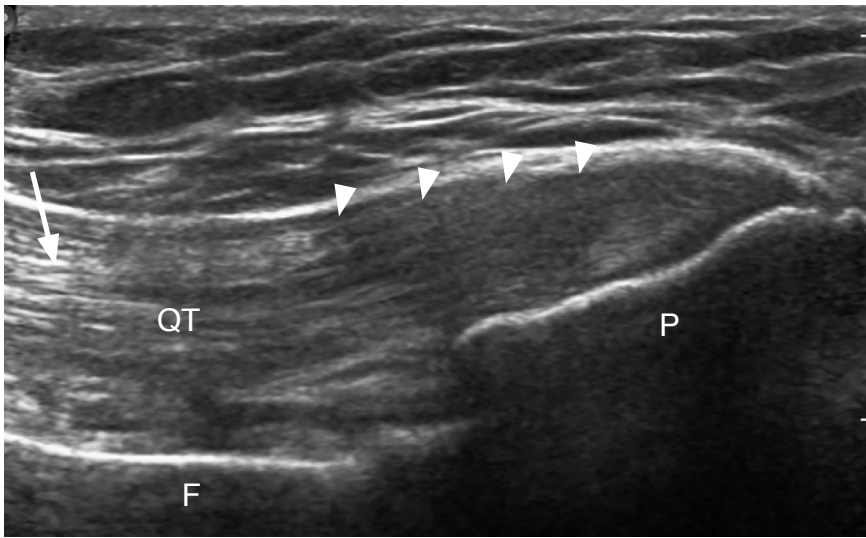

Fig. 6. A 60-year-old woman with quadriceps tendinosis. Ultrasonography of the anterior knee long axis to quadriceps tendon (QT) shows a thickened and hypoechoic tendon (arrowheads). Proximally, a normal fibrillar pattern is seen (arrow). P, patella; F, femur.

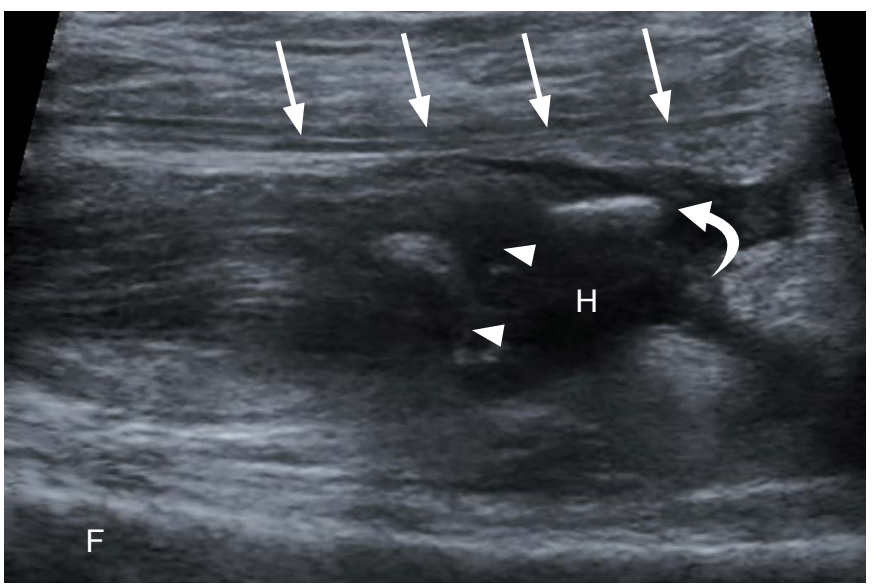

Fig. 7. A 27-year-old man with partial quadriceps tear. Ultrasonography of the anterior knee long axis to quadriceps tendon shows discontinuity and retraction of the mid deep tendon fibers (arrowheads). A linear hyperechoic fracture fragment (curved arrow) and hematoma $(H)$ are seen. The superficial rectus femoris tendon fibers remain intact (arrows). F, femur.

the rectus femoris, the middle layer is comprised of the combined vastus medialis and vastus lateralis, and the deep layer arises from the vastus intermedius $[17,19,20]$. While the majority of the quadriceps tendon inserts at the anterosuperior patella, superficial rectus femoris fibers continue, attached to the anterior surface of the patella and join with the patellar tendon, referred to as the prepatellar quadriceps continuation [21].

Similar to previous descriptions, tendinosis is manifested as a hypoechoic and possibly thickened tendon (Fig. 6). While color Doppler may reveal hyperemia, the term tendinosis is used

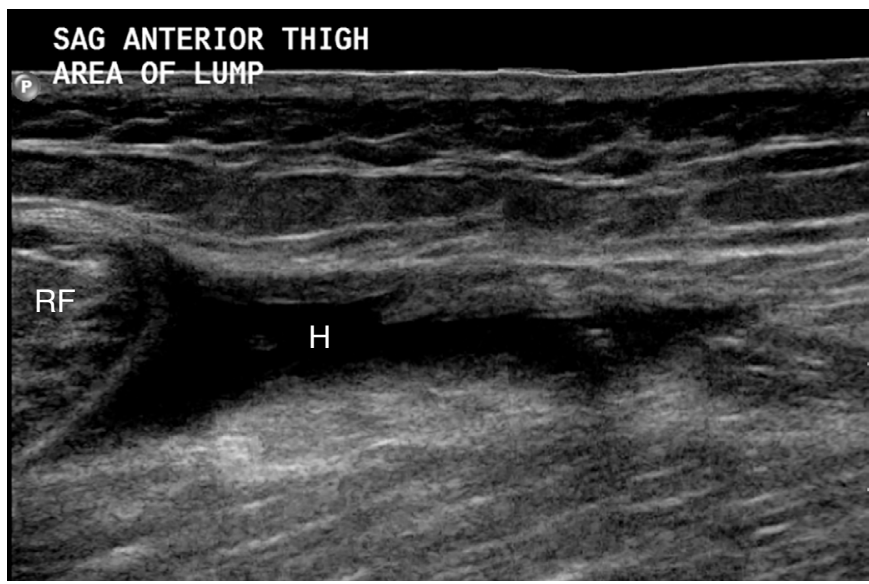

Fig. 8. A 54-year-old man with full-thickness rectus referred for a "mass." Long-axis ultrasonography of the anterior thigh demonstrates complete disruption of the rectus femoris tendon with retraction of the rectus femoris muscle (RF) and interposed hypoechoic hematoma $(\mathrm{H})$. Right side of image is distal.

instead of tendinitis as hyperemia is due to neovascularity and not inflammation. Anechoic clefts at the patellar insertion representing tears can involve one, two, or all three layers (Fig. 7). Full thickness ruptures often occur $1-2 \mathrm{~cm}$ proximal to the insertion at the avascular zone; however, injury at the insertion with resultant cortical irregularity or even an echogenic, retracted and shadowing avulsion fracture may be present $[19,20]$. While ruptured tendon typically retracts with wavy fibers, in subtle or equivocal cases, dynamic maneuvers where the leg is passively flexed and extended, or where the patella is manually translated inferiorly, can elucidate whether proximal intact fibers move with the patella $[19,20]$. Separation of the prepatellar quadriceps continuation from the anterior patellar cortex with intervening hypoechoic fluid has also been described in the setting of anterior knee pain [21]. An isolated tear of the distal rectus femoris may retract and present much later after the injury as a palpable "pseudotumor" representing the retracted tendon stump (Fig. 8).

\section{Jumper's Knee}

Patellar tendinopathy resulting in anterior knee pain is colloquially referred to as "jumper's knee." Similar to quadriceps tendon injury, and as the name would suggest, this affects individuals who play sports requiring repetitive jumping $[22,23]$. True tendon inflammation is not present and therefore the term tendinitis is not appropriate. Abnormal imaging findings include hypoechoic tendinosis, possible anechoic interstitial tears, and hyperemia resulting from neovascularity, which correlates with the severity of patient symptoms (Fig. 9). The deep aspect of the proximal patellar 


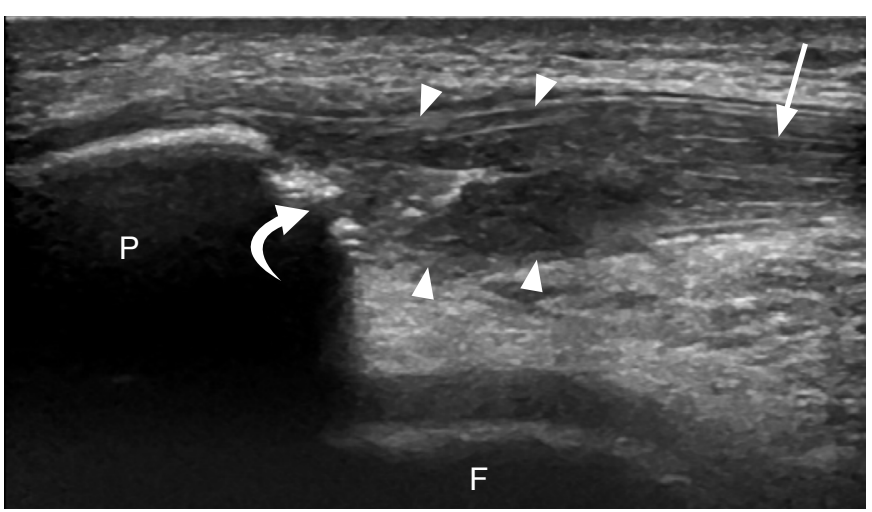

A

Fig. 9. A 13-year-old boy with patellar tendinosis (jumper's knee).

A, B. Long-axis (A) and short-axis (B) ultrasonography demonstrates a thickened, hypoechoic, and hyperemic central patellar tendon (arrowheads) at its origin with irregularity of the inferior patellar pole cortex (curved arrow). Normal fibrillar tendon is seen distally and laterally (arrow). P, patella; F, femoral condyle.

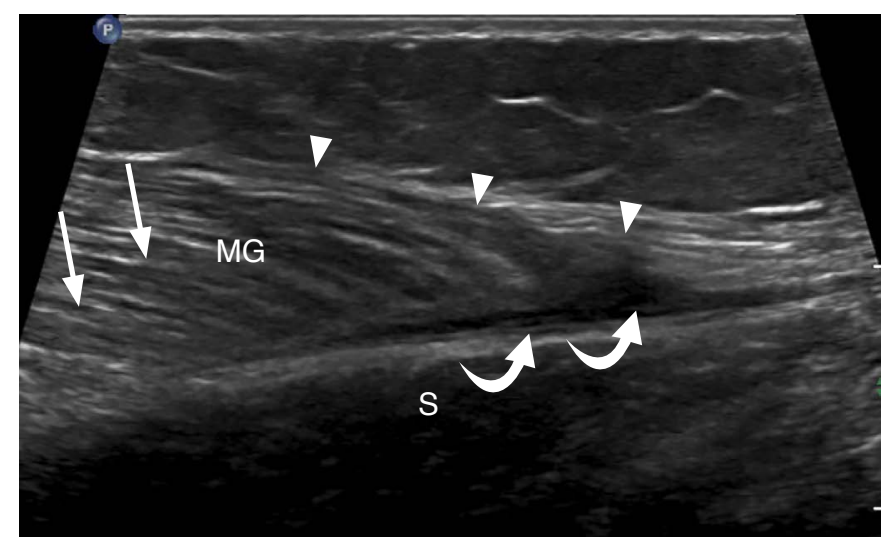

Fig. 10. A 30-year-old woman with medial head gastrocnemius tear (tennis leg). Ultrasonography of the calf long axis to the distal medial head of the gastrocnemius (MG) demonstrates an irregular and hypoechoic distal myotendinous junction (arrowheads) with a small hypoechoic hematoma (curved arrows) between the MG and soleus (S). Compare to the normal muscle appearance (arrows).

tendon is the most common site of involvement $[17,22,23]$.

\section{Ankle and Leg}

\section{Medial Gastrocnemius Tear}

Tears of the medial head of the gastrocnemius, referred to as tennis leg, most commonly affect middle-aged athletes who note acute pain in the mid-calf during simultaneous active ankle plantar flexion and knee extension [24]. Recent studies have demonstrated that medial head of gastrocnemius tears most often occur at the distal myotendinous junction and are more common than plantaris tendon

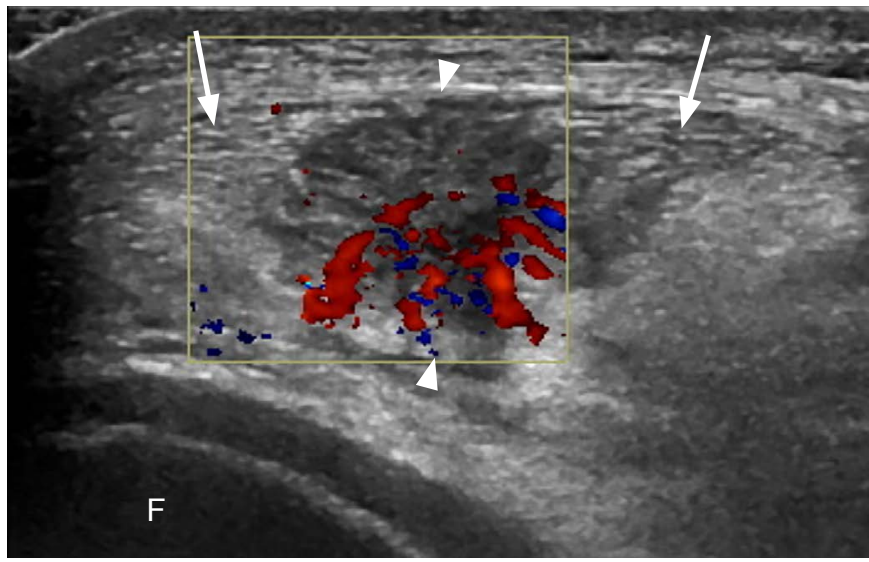

B

rupture, which was previously hypothesized to be responsible for this clinical syndrome [25]. In the evaluation of symptoms suspicious for tennis leg, the ultrasound operator should consider an alternative diagnosis of deep venous thrombosis, which can also present with calf symptoms.

The medial head of the gastrocnemius is best evaluated in the longitudinal orientation. Ultrasound characteristics of a gastrocnemius tear include disruption of the normal alternating linear hyperechoic and hypoechoic appearance at the distal myotendinous junction, where hypoechoic hemorrhage collection replaces the normal tapered appearance of the distal aspect (Fig. 10) [25]. Larger tears are characterized by retraction and heterogeneous fluid extending proximally, between the muscle bellies of the medial head of the gastrocnemius and the soleus. As the tear heals, fibrous tissue interposed between the medial gastrocnemius and soleus can be identified as an area of heterogeneous increased echogenicity $[24,26]$. Treatment for tennis leg is typically conservative.

\section{Plantaris Rupture}

Patients presenting with a ruptured plantaris tendon describe the sudden onset of calf pain, which feels as though they have been kicked or had direct impact from a projectile, similar to patients injuring their medial head of gastrocnemius $[25,27]$. The plantaris muscle origin is along the posterior superior aspect of the lateral femoral condyle, with the muscle extending inferiorly and medially along the posterior knee. The long and thin plantaris tendon, located between the soleus and gastrocnemius medial head muscle bellies, terminates either on the calcaneus adjacent to the posterior medial aspect of the Achilles tendon, or on the medial Achilles tendon 
itself $[27,28]$. The plantaris may be absent in up to $7 \%-10 \%$ of the population [28]. On ultrasound, plantaris rupture is diagnosed by absence of the normal plantaris tendon with heterogeneously hypoechoic tubular fluid representing hematoma in its expected location between the soleus and medial head of the gastrocnemius muscle bellies (Fig. 11) $[25,27,28]$.

\section{Achilles Tendon Injury}

Achilles tendon injury usually affects athletes in the third to sixth decades of life and results from sudden or repetitive resisted dorsiflexion [29]. Anatomically, the tendon receives contributions from the medial and lateral heads of the gastrocnemius, as well

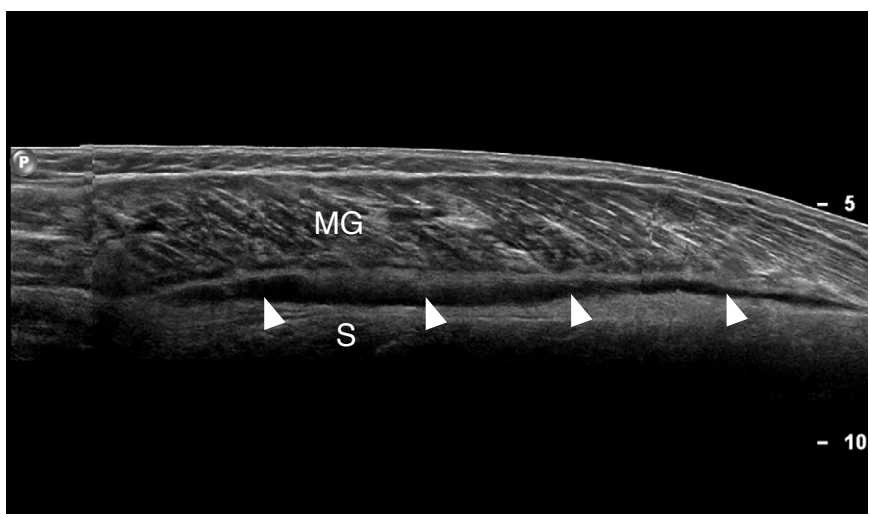

Fig. 11. A 32-year-old woman with plantaris rupture. Ultrasonography of the calf long axis to the medial head of gastrocnemius (MG) demonstrates a heterogeneous, hypoechoic hematoma (arrowheads) tracking between the medial head gastrocnemius and soleus (S). No plantaris tendon is identified.

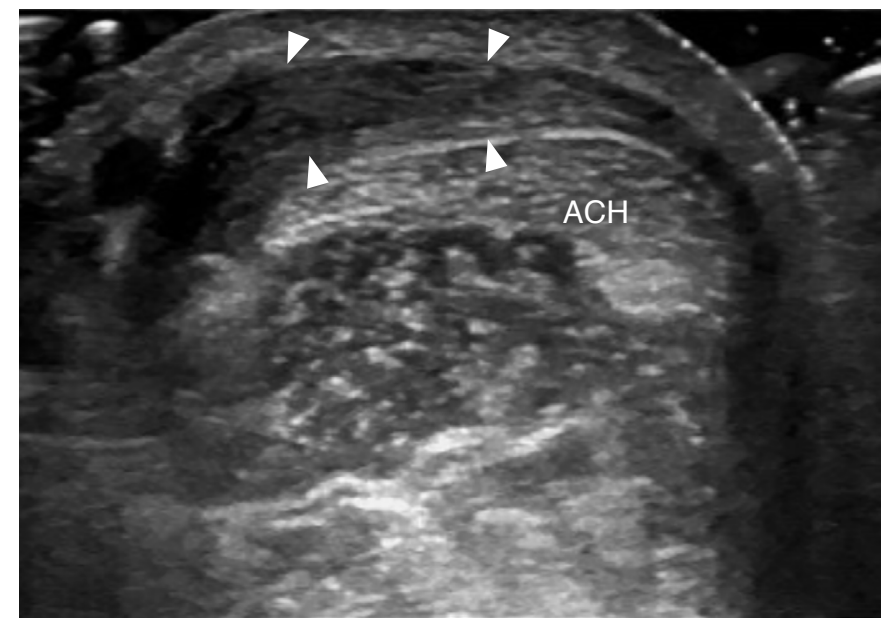

A

Fig. 12. A 44-year-old woman with Achilles paratenonitis.

A, B. Ultrasonography of short axis (A) and long axis (B) to Achilles tendon (ACH) show thickened and heterogeneously hypoechoic paratenon (arrowheads).

e-ultrasonography.org

Ultrasonography 37(3), July 2018 
spurring at the posterosuperior calcaneal tuberosity may be incidental or the result of insertional tendinosis. Hyperemia on color Doppler and distention of the retrocalcaneal bursa (greater than 2.5 $\mathrm{mm}$ ) can further support this latter diagnosis [30]. Myotendinous junction injury results in disorganization of the normally parallel echogenic muscle fibers with adjacent hypoechoic edema [33].

With a full-thickness complete Achilles tendon tear, there retraction of the proximal tendon fibers and a positive Thompson test, where squeezing the calf does not result in the normal plantarflexion of the foot [36]. On ultrasound, retracted tendon fibers are wavy and irregular with heterogeneously hypoechoic intervening fluid. It is important to document both the stump quality as well as the residual gap between the tendon stumps during dorsiflexion, as this helps guide clinical decision-making regarding surgical versus non-surgical management (Fig. 14). Full-thickness tears present as tendon discontinuity and retraction during dynamic evaluation with passive plantar flexion and dorsiflexion [30]. The use of dynamic imaging that demonstrates tendon discontinuity is an important component of the ultrasound examination to achieve high accuracy in full-thickness tear diagnosis. The plantaris almost uniformly remains intact and should not be misinterpreted as intact Achilles tendon fibers.

\section{Peroneal Tendinopathy}

A frequent cause of posterolateral ankle pain, the peroneal tendons are prone to overuse injury [37]. While the peroneus longus, arising proximally from the fibula and tibia, has its myotendinous junction well before it reaches the ankle, the peroneus brevis muscle, originating from the distal fibula, tapers distally to the lateral malleolus where the tendons pass posteriorly in the retromalleolar groove. The peroneus brevis is typically in contact with the fibula, between the bone and the peroneus longus, which likely accounts for its predisposition to injury at this location. The tendons are held in place as they pass behind and distal to the lateral malleolus by

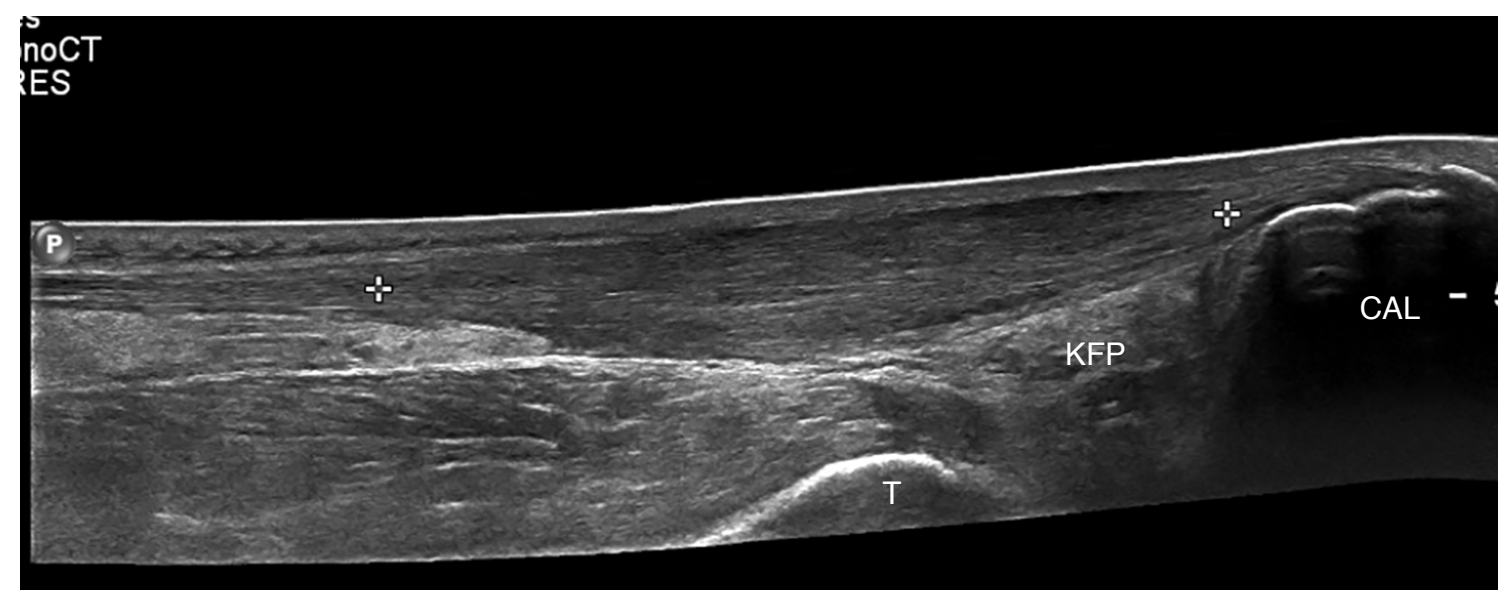

A

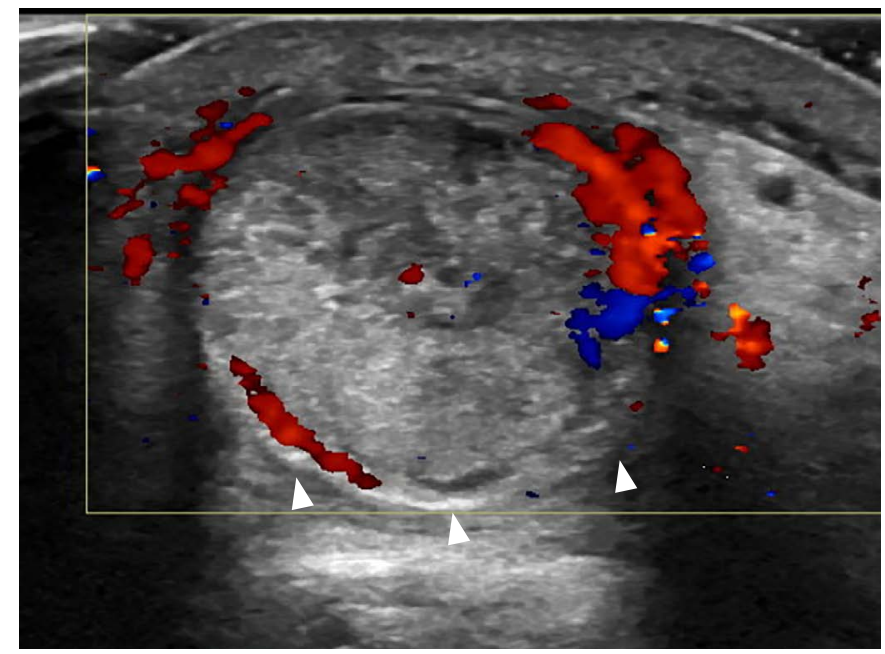

B
Fig. 13. A 61-year-old woman with Achilles tendinosis.

$A, B$. Ultrasonography of long axis (A) and short axis (B) to Achilles tendon demonstrates a long segment of thickened tendon (between the calipers). This is the watershed zone approximately 2-6 cm proximal to the insertion on the posterior calcaneus (CAL). Hyperemia representing paratenonitis (arrowhead) is also noted. T, tibia; KFP, Kager fat pad. 


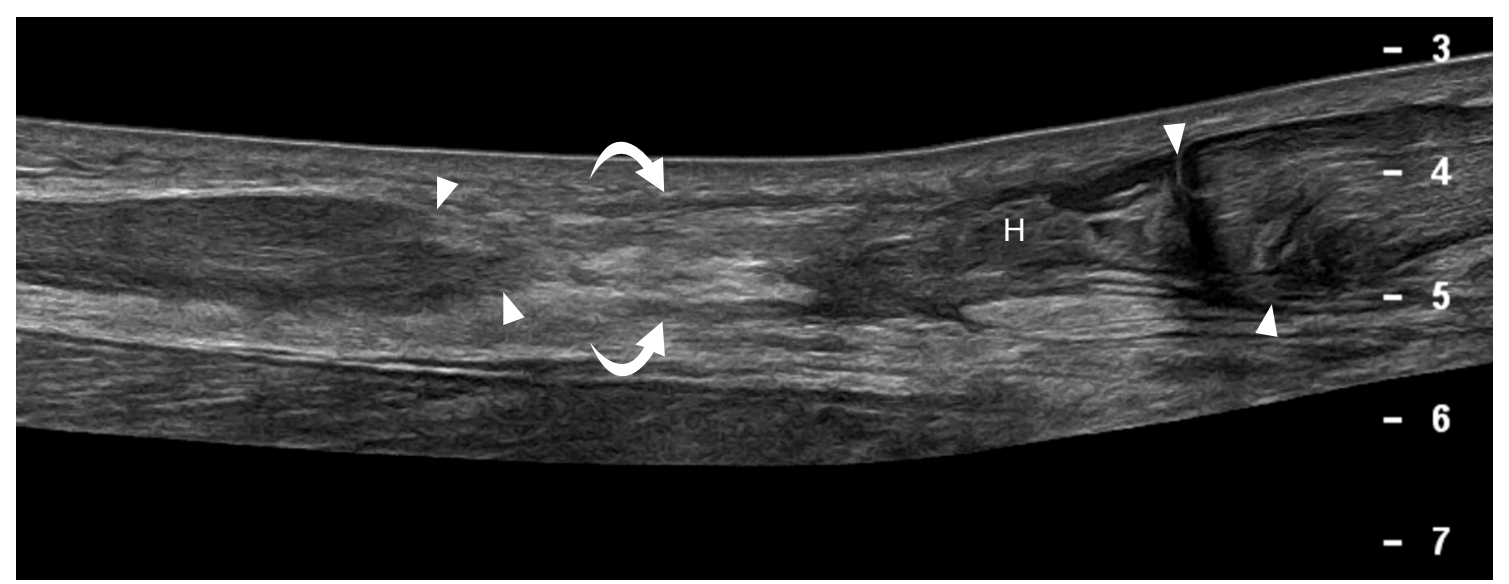

Fig. 14. A 50-year-old woman with full-thickness Achilles tendon tear. Ultrasonography of long axis to Achilles tendon demonstrates a fullthickness tear with retraction of proximal and distal edges (arrowheads). Note the interposed heterogeneous hematoma $(\mathrm{H})$ and echogenic fat (curved arrows).

the superior and inferior peroneal retinacula. Distally, the tendons course anteriorly, on either side of a bony excrescence from the calcaneus, termed the peroneal tubercle, until the brevis inserts at the base of the fifth metatarsal and the longus, having curved along the plantar aspect of the foot, inserts at the base of the first metatarsal and medial cuneiform $[37,38]$.

At ultrasound, peroneal tendinosis appears as hypoechogenicity with possible tendon enlargement. Anechoic clefts indicate superimposed interstitial tearing. An anechoic cleft that extends to the surface of the tendon, commonly the peroneus brevis, is termed a longitudinal split tear (Fig. 15). A full-thickness complete tear presents as tendon discontinuity with retraction. Tenosynovitis can vary from anechoic fluid distention of the tendon sheath to mixedechogenicity but predominantly hypoechoic hypervascular synovitis $[37,38]$.

\section{Peroneal Tendon Subluxation and Dislocation}

Abnormal movement of the peroneal tendons may result in snapping, pain, and tendon injury, and is best evaluated with ultrasound during dynamic imaging with ankle dorsiflexion and eversion $[39,40]$. Peroneal tendon subluxation and dislocation are the sequelae of injury to the superior peroneal retinaculum, which normally holds the peroneal tendons in place along the posterior fibula in the retromalleolar groove. When injured or torn, the retinaculum may appear hypoechoic or discontinuous, with or without an avulsion fragment retracted from its insertion on the fibula [40]. During dorsiflexion and eversion stress maneuvers, one or both peroneal tendons may partially move anterior and lateral from their normal location (subluxation) or completely displace (dislocation), and return during rest, predisposing to tenosynovitis

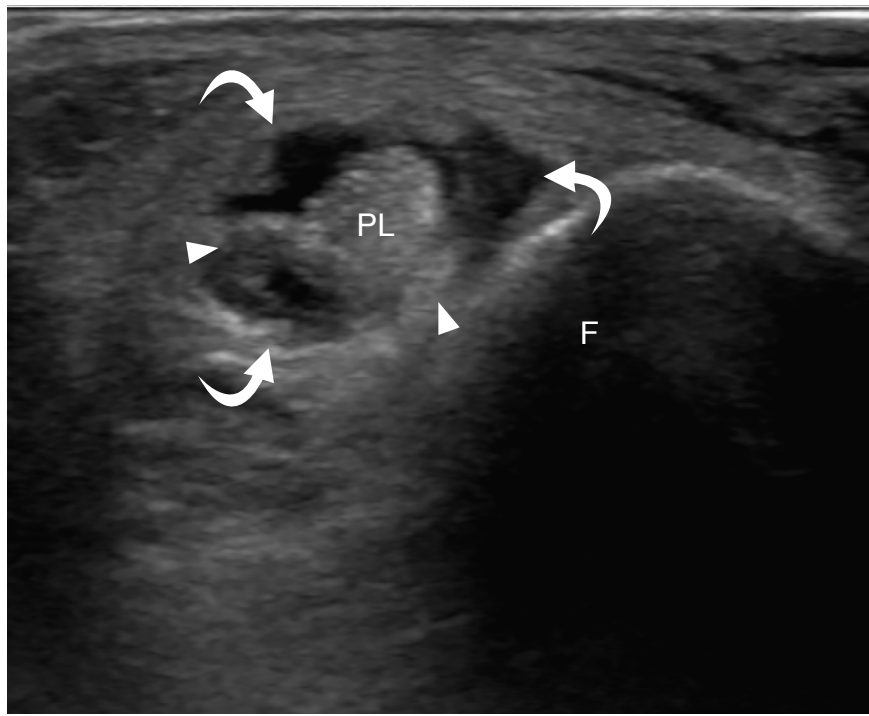

Fig. 15. A 59-year-old woman with peroneus brevis longitudinal split tear. Ultrasonography of short axis to peroneus longus tendon (PL) at the level of the distal fibula (F) demonstrates a longitudinal split tear of the peroneus brevis with two separate bundles (arrowheads) with hypoechoic tenosynovitis (curved arrows).

and tendon tear (Fig. 16) [39].

Intrasheath peroneal tendon subluxation occurs when the peroneus longus and brevis tendons abnormally alternate positions between themselves producing a snapping sensation, while remaining deep to an intact superior retinaculum. This condition may be associated with a longitudinal split tear of the peroneus brevis, where the tendon of the peroneus longus moves in between the two brevis tendon bundles during stress. Intrasheath subluxation is associated with an abnormally convex posterior border of the 


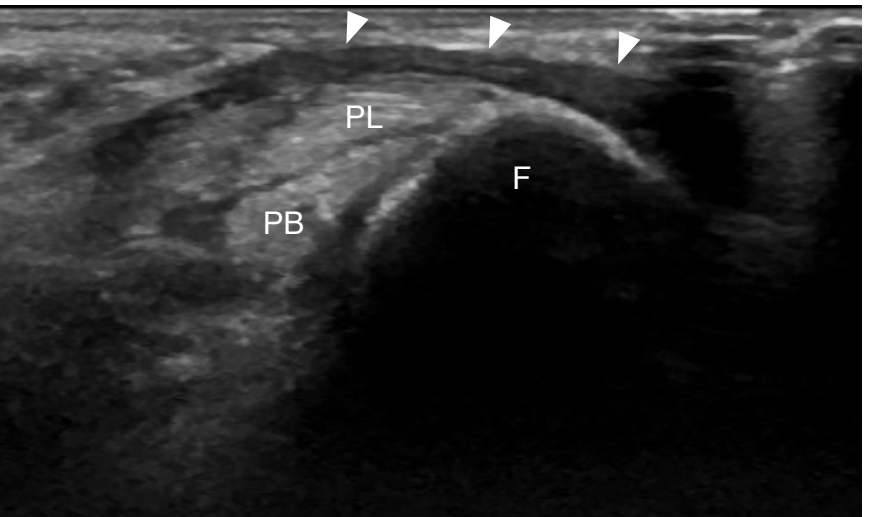

A

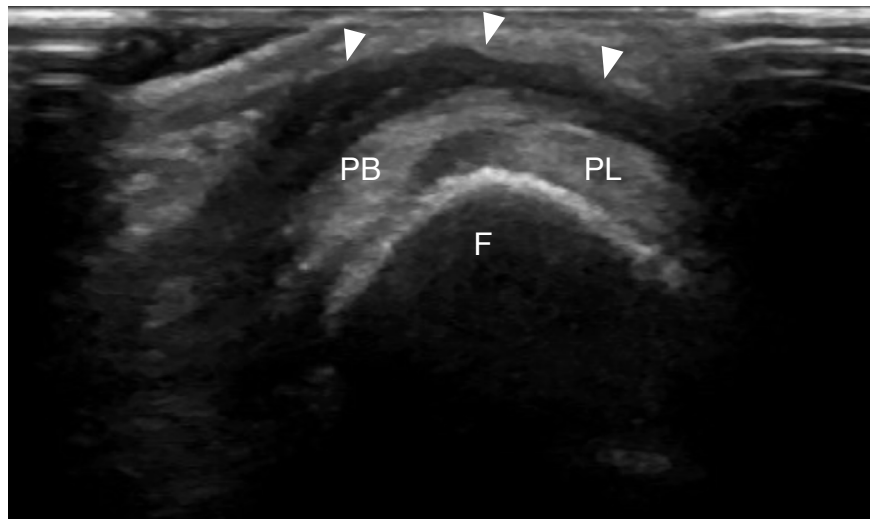

B

Fig. 16. A 57-year-old woman with peroneal tendon subluxation/dislocation.

A, B. Transverse ultrasonography at the distal fibula (F) at rest (A) and during dorsiflexion (B) and eversion demonstrate an abnormally thickened superior peroneal retinaculum (arrowheads) which is stripped from the fibula. This allows anterior dislocation of the peroneus longus (PL) and subluxation of the peroneus brevis $(\mathrm{PB})$ in $\mathrm{B}$.

lateral malleolus, a low lying brevis muscle where the myotendinous junction extends distal to the lateral malleolus, or an accessory peroneus quartus muscle and tendon at this level [40].

\section{Anterior Talofibular Ligament Tear}

One of the most biomechanically important ligaments of the ankle, the anterior talofibular ligament (ATFL) is also the most frequently and often first to be injured, and is the result of abnormal inversion stress [41]. In order to identify the ligament, either palpate or locate the extreme distal tip of the fibula with ultrasound in the transverse plane. Then, slide the transducer anteriorly and slightly superiorly until both the fibula and talus are in the field of view. The obliquely oriented ATFL may be slightly hypoechoic from anisotropy, but altering the probe angle with a heel-toe maneuver should reveal the normal echogenic, fibrillar pattern [42].

Injuries to the ATFL produce imaging features similar to other ligamentous injuries. In an acute partial tear, the ligament is hypoechoic with some intact fibers remaining. In an acute full thickness tear, the fibers are discontinuous or absent, replaced by heterogeneous hematoma (Fig. 17). Avulsion fractures are identified as echogenic foci adjacent to sites of ligamentous insertion. Dynamic imaging with an anterior drawer test can help distinguish between a partial or full thickness tear; talus motion can assist in visualizing intact ligament fibers, and asymmetric anterior talus subluxation suggests ligament failure. This maneuver is completed with the patient prone, the transducer placed over the ATFL, and stress placed on the posterior calcaneus [41]. With a chronic tear, the ligament may be absent, thinned, or thickened, but the patient should not have associated symptoms with transducer pressure [43].

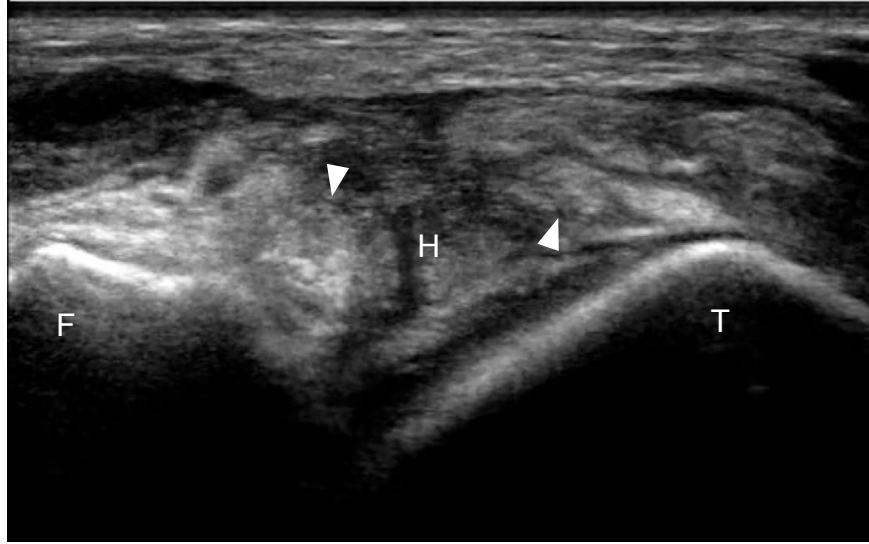

Fig. 17. A 17-year-old girl with acute anterior talofibular ligament tear. Ultrasonography over the anterolateral ankle in the transverse plane demonstrates complete disruption of the anterior talofibular ligament with irregular and frayed tibial and fibular stumps (arrowheads) and interposed heterogeneous hypoechoic hematoma (H). T, talus; F, fibula.

With suspected lateral ankle ligament injury, the calcaneofibular ligament should also be assessed, which typically tears in succession after the ATFL tears, appearing as a hypoechoic and thickened ligament adjacent to the calcaneal body (Fig. 18).

\section{High Ankle Sprain}

During extreme ankle eversion, the first of the ankle syndesmotic ligaments to be injured is the anterior inferior tibiofibular ligament (AiTFL). This ligament is identified by first orienting the transducer over the anterior talofibular ligament, then rotating the talar (medial) side of the probe superiorly so that the probe is oblique and the 


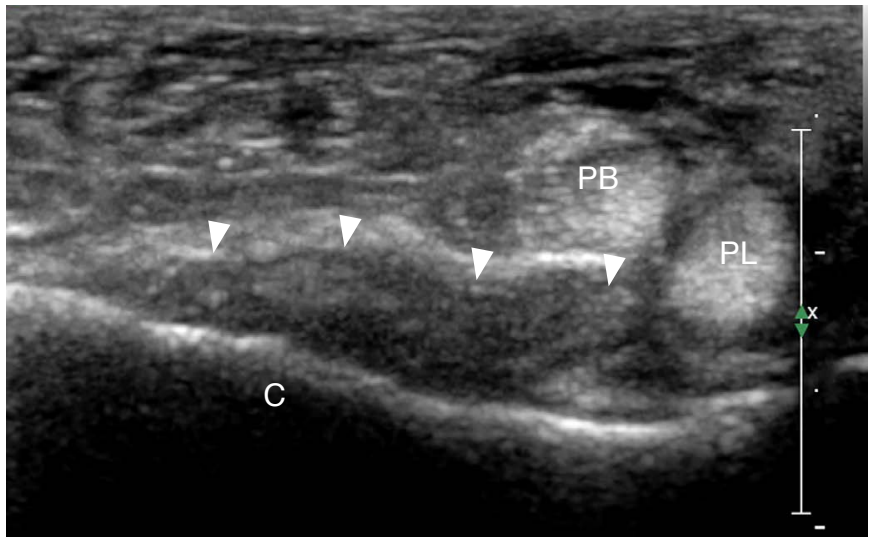

Fig. 18. A 17-year-old boy with acute calcaneofibular ligament tear. Ultrasonography of lateral ankle in the oblique coronal plane demonstrates a thickened, hypoechoic and irregular calcaneofibular ligament (arrowheads) consistent with tear. PL, peroneus longus; PB, peroneus brevis; $\mathrm{C}$, calcaneus.

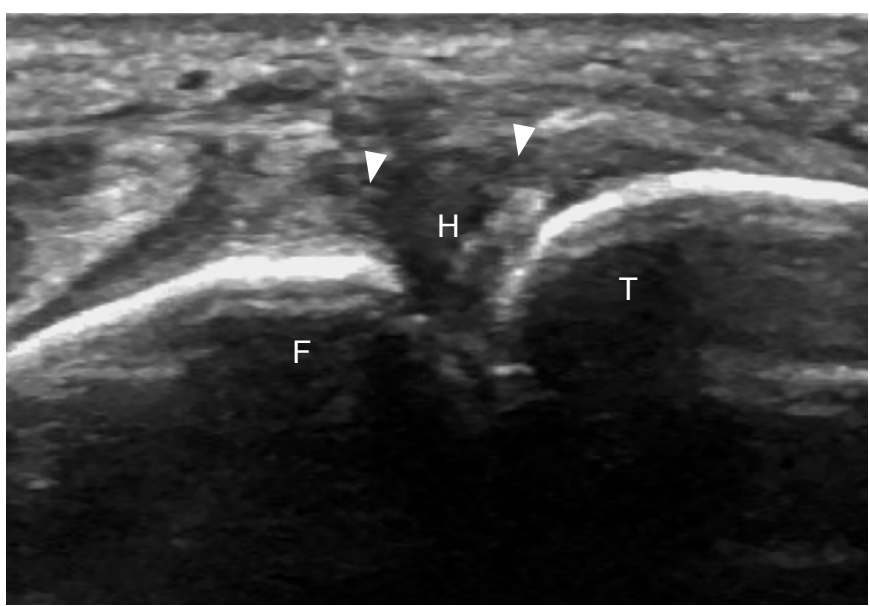

Fig. 19. A 49-year-old woman with acute anterior inferior tibiofibular ligament tear. Ultrasonography in the transverse oblique plane at the level of the distal tibia demonstrates complete disruption of the anterior tibiofibular ligament with irregularity of the remaining stumps (arrowheads) and heterogeneous, hypoechoic intervening fluid (H). T, tibia; F, fibula.

fibrillar ligament comes into the field of view. The resulting imaging plane should be similar to that for the calcaneofibular ligament but on the tibial side of the fibula. Ligament tears will appear as hypoechoic thickening or ligament discontinuity (Fig. 19) [44]. Because the AiTFL is the first ligament to be injured in eversion, if this is intact, interosseous membrane injury is uncommon [45].

If the AiTFL is injured, however, injury to the interosseous membrane, commonly referred to as a "high ankle sprain," should be excluded as this may lead to delayed healing and recovery, instability, and accelerated degenerative change [46].
The interosseous membrane is identified as a thin echogenic linear structure between the tibia and fibula and may be discontinuous, thickened, and hypoechoic when injured. Dynamic imaging can be a helpful tool in evaluating integrity, assessed by measuring the tibiofibular clear space $1 \mathrm{~cm}$ proximal to the joint line. Normally there should be minimal difference in the measurements of the tibiofibular clear space between internal and external rotation at the ankle (usually a difference of less than $2 \mathrm{~mm}$, but up to $5 \mathrm{~mm}$ ). Significant widening of the clear space during external rotation indicates injury to the interosseous membrane. Injuries may propagate proximally through the interosseous membrane and exit through the proximal fibula, resulting in a Maisonneuve fracture, by definition, which can be identified as a cortical step off $[44,45]$. In the setting of clinical suspicion, radiographs of the proximal fibula are often helpful for confirmation of a Maisonneuve fracture.

\section{Muscle Hernia}

Muscle herniations often present a diagnostic dilemma, as patients may be referred for pain, cosmetic mass, or concern for a tumor. A muscle hernia occurs when normal muscle protrudes from its normal anatomic compartment through a defect in the overlying fascia and into the subcutaneous fat. Eighty-nine percent of muscle hernias occur in the lower extremities, most frequently involving the tibialis anterior muscle [47], but also often involving the peroneal, gastrocnemius, soleus, quadriceps and erector spinae muscles $[47,48]$. Pain associated with this entity may be related to transient muscle strangulation or superficial nerve entrapment; however some have described a correlation with chronic exertional compartment syndrome, which may serve an alternative etiology for pain [47]. Treatment depends on symptomatology and may range from reassurance that the lesion is benign, to activity modification, compression stockings and non-steroidal anti-inflammatory medications to surgical intervention with fasciotomy [47].

During ultrasound evaluation, a few technical considerations can improve diagnostic accuracy. The mass should be palpated by hand and marked with a pen, as the abnormality may be difficult to palpate with the ultrasound probe; liberal use of gel as a standoff enables the use of light pressure ensuring that the hernia is not reduced or obscured, and dynamic evaluation during muscle contraction or with the patient standing can accentuate the hernia [49].

Normal fascia should appear as a thin echogenic line immediately superficial to the muscle. A muscle hernia is diagnosed when imaging demonstrates a defect in the echogenic fascia with a portion of the underlying muscle protruding through and over the fascia, often assuming a mushroom shape (Fig. 20). The herniated muscle may be slightly less echogenic than adjacent normal muscle 


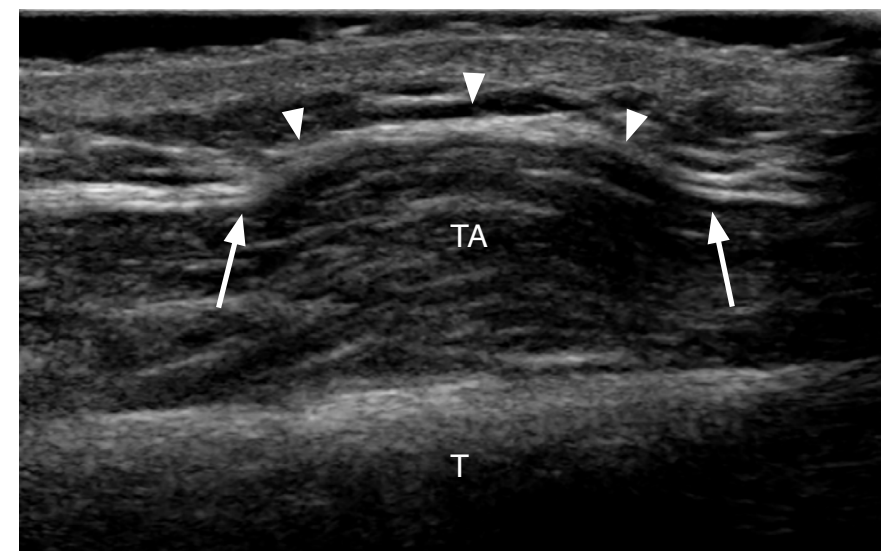

A

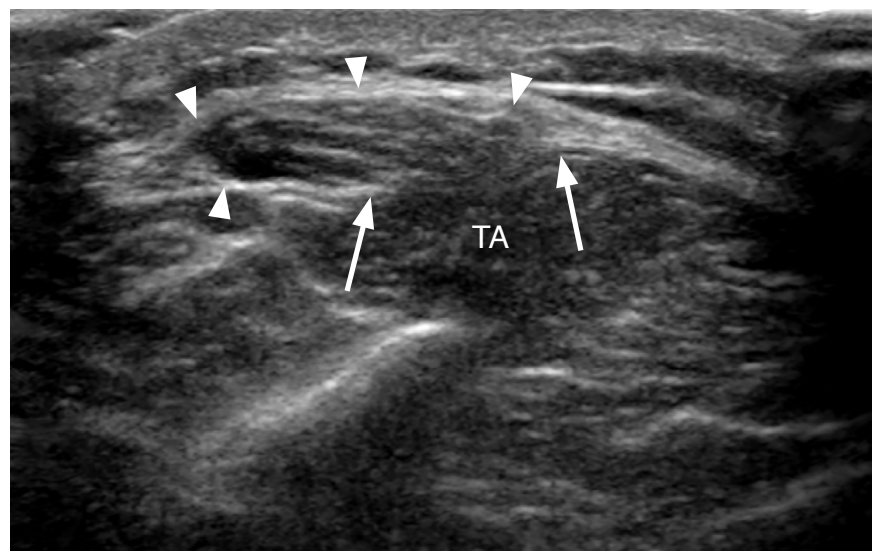

B

Fig. 20. A 21-year-old man with tibialis anterior muscle hernia.

A, B. Longitudinal (A) and transverse (B) ultrasonography over the anterior leg demonstrates a defect in the deep fascia (arrows) overlying the tibialis anterior (TA). Muscle fibers are seen herniating through the defect into the overlying subcutaneus fat (arrowheads). T, tibia.

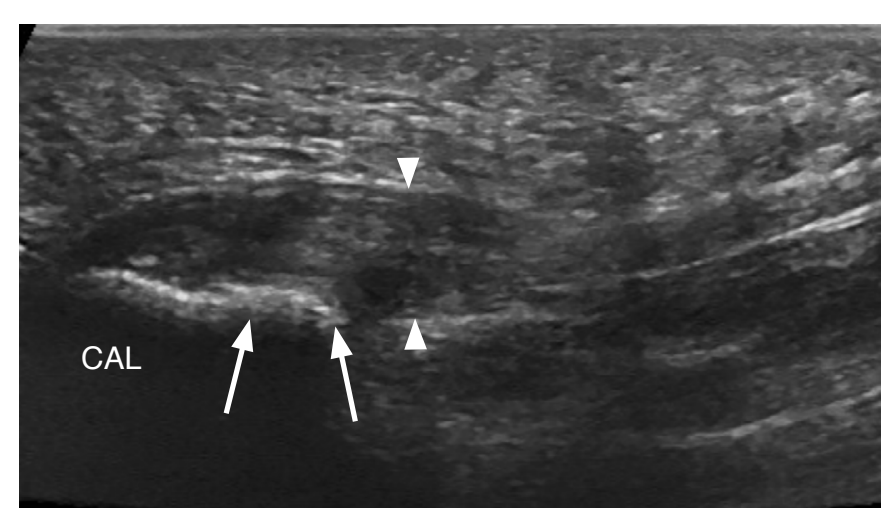

Fig. 21. A 53-year-old man with plantar fasciopathy. Ultrasonography of the plantar foot long axis to the plantar fascia shows abnormally thickened and hypoechoic plantar fascia (arrowheads). A small plantar calcaneal spur is seen (arrows). CAL, calcaneus.

and may be secondary to anisotropy or atrophy $[47,48]$. Blood vessels are sometimes noted traversing the fascia at the site of the defect and have been hypothesized as potential cause of focal fascial weakness [49].

\section{Foot}

\section{Plantar Fasciopathy}

Distance running may result in chronic and sometimes exquisite heel pain. One etiology for this type of pain is repetitive microtrauma to the plantar aponeurosis, resulting in plantar fasciopathy [50]. Inflammation is absent in this condition therefore the term fasciitis should not be used. Originating from the calcaneal tuberosity, the plantar aponeurosis is a thick fibrous band that is divided into three cords. The central cord is the thickest and most often injured, enveloping the flexor digitorum brevis muscle and dividing anteriorly into five fascicles that insert distally onto the skin, flexor tendon sheaths, and plantar plates of the phalanges [51]. The medial and lateral cords envelop the abductor hallucis brevis and abductor digiti minimi, respectively, and are rarely injured.

Ultrasound features of plantar fasciopathy include a thickened (greater than $4 \mathrm{~mm}$ ) and hypoechoic aponeurosis close to its calcaneal attachment (Fig. 21) [51]. Anechoic clefts may be seen in interstitial tearing, and, more rarely, complete tears demonstrate retraction of the discontinuous aponeurotic fibers with intervening heterogeneous hematoma. Hyperemia may be present on color Doppler evaluation and the patient often exhibits focal tenderness over the abnormality. A bony enthesopathic spur at the calcaneal tuberosity may be present in symptomatic or asymptomatic individuals $[50,51]$. Contrary to popular belief, recent studies have demonstrated no correlation between fascial thickness and degree of symptoms. Biconcavity, however, is a feature that has been associated with a diminished response to supportive therapy [52].

\section{Turf Toe}

Turf toe is an injury at the first metatarsophalangeal (MTP) joint that results from an axial load while the joint is in hyperextension. This typically affects American football players who play on hard, artificial surfaces with flexible shoes, resulting in injury to the supporting structures of the joint [53]. The anatomy of the first MTP capsuloligamentous complex consists of a cartilaginous plantar plate, two sesamoid bones, several ligaments (intersesamoidal, metatarsosesamoidal, sesamoid phalangeal, collateral), and a joint capsule, with support provided by the tendons of the flexor hallucis 


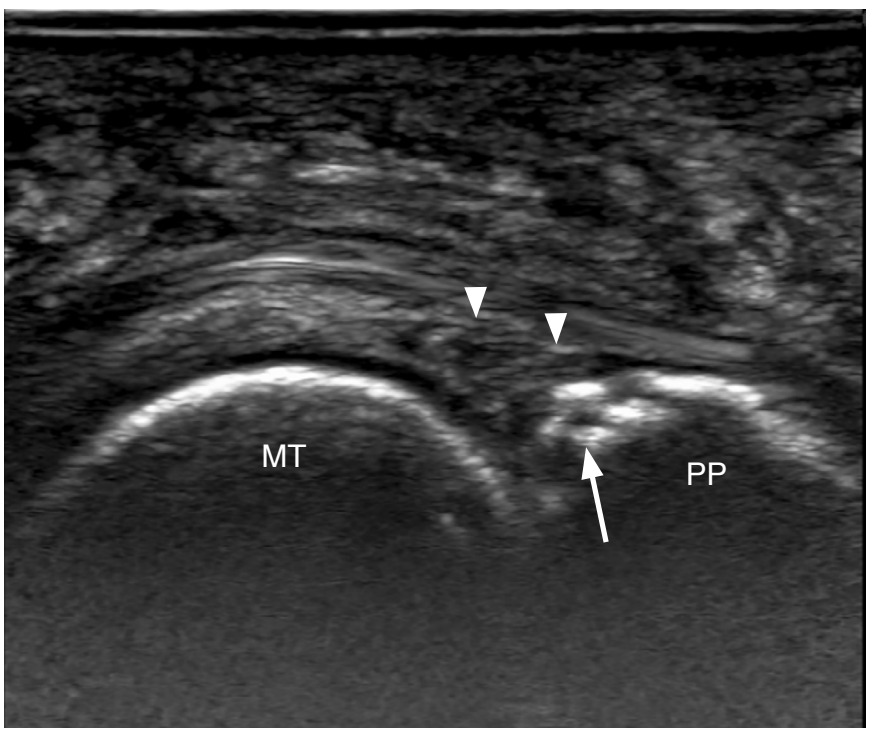

Fig. 22. A 57-year-old woman with plantar plate injury. Longitudinal ultrasonography at the plantar aspect of the second metatarsophalangeal joint demonstrates abnormal heterogeneous and hypoechoic appearance of the plantar plate (arrowheads) with cortical irregularity at its insertion on the proximal phalanx (arrow). MT, metatarsal; PP, proximal phalanx.

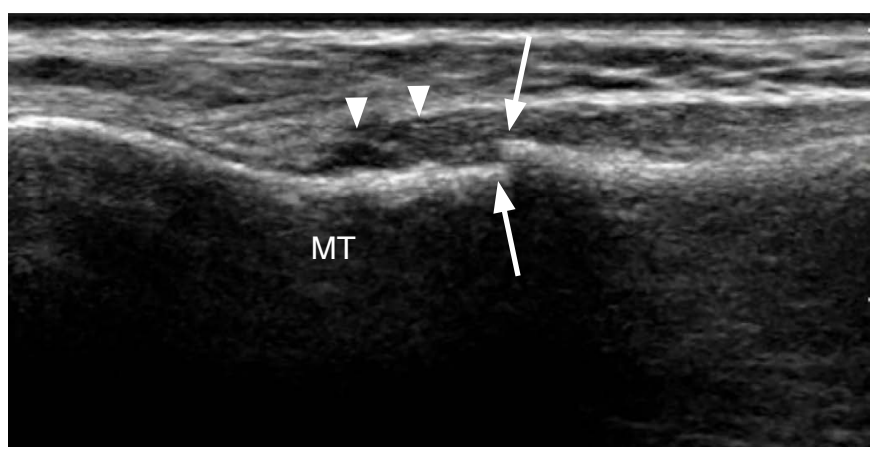

Fig. 23. A 21-year-old woman with metatarsal stress fracture. Longitudinal ultrasonography over the fourth metatarsal (MT) demonstrates a cortical step off (arrows) and overlying hypoechoic edema (arrowheads).

brevis and longus, and abductor and adductor hallucis [53,54]. The plantar plate originates at the metatarsal neck and inserts at the plantar aspect of the base of the proximal phalanx associated with the joint capsule $[53,54]$.

Ultrasound in the setting of turf toe may demonstrate hypoechoic soft tissue edema, a thickened and hypoechoic plantar plate, ligament thickening or discontinuity, a hypoechoic cleft between the plantar plate and proximal phalanx, or an echogenic avulsion fracture from the plantar plate insertion at the base of the proximal phalanx (Fig. 22). Although the sensitivity of ultrasound in the diagnosis of plantar plate tear is $96 \%$, a heterogeneous appearance may be seen as an asymptomatic finding, which likely accounts for its poor specificity [55]. On physical exam there may be ecchymosis at the volar MTP joint and focal pain. A drawer test, where the MTP is stressed dorsally, is positive when there is excessive dorsal translation of the proximal phalanx and supports the diagnosis of turf toe $[53,54,56]$.

\section{Stress Injury and Fracture}

Fatigue-type stress injury, resulting from excessive stress on otherwise healthy bone, most often affects the second and third metatarsal shafts in a ballet dancer, running athlete, or military personnel, but can involve any metatarsal $[53,57]$. Often the history will reveal a sudden recent increase in activity level. Manifesting as vague forefoot pain during exercise, stress injury follows a predictable pattern, as repetitive mechanical forces exceed the ability of the bone to grow and repair itself, resulting in microfractures [57]. Periostitis and periosteal new bone formation are seen in the least severe cases, followed by bone marrow edema, then incomplete fracturing and eventually a completed fracture.

Although it is not feasible to evaluate all of the osseous structures on ultrasound, a targeted evaluation at the patient's area of point tenderness may reveal findings supportive of this diagnosis. Hypoechoic soft tissues indicating swelling, cortical irregularity, and periosteal thickening may be seen at the site of stress injury, while a frank cortical step-off is diagnostic of a fracture (Fig. 23) [58,59].

\section{Conclusion}

As clinical applications for ultrasound in musculoskeletal care continue to expand, it has become increasingly important to have an understanding of common athletic injuries in the lower extremities and their sonographic appearances. This article highlights several such injuries in an effort to review basic anatomic relationships, pathologic appearances, and the importance of the dynamic ultrasound evaluation.

ORCID: Nathaniel B. Meyer: http://orcid.org/0000-0002-9600-0288; Jon A. Jacobson: http://orcid.org/0000-0002-3676-6628; Vivek Kalia: http://orcid.org/0000-00025061-1702

\section{Conflict of Interest}

No potential conflict of interest relevant to this article was reported.

\section{References}

1. Jacobson JA. Fundamentals of musculoskeletal ultrasound. 
Philadelphia, PA: Elsevier, 2018.

2. Hegazi TM, Belair JA, McCarthy EJ, Roedl JB, Morrison WB. Sports injuries about the hip: what the radiologist should know. Radiographics 2016;36:1717-1745.

3. Bureau NJ. Sonographic evaluation of snapping hip syndrome. J Ultrasound Med 2013;32:895-900.

4. Guillin R, Cardinal E, Bureau NJ. Sonographic anatomy and dynamic study of the normal iliopsoas musculotendinous junction. Eur Radiol 2009;19:995-1001.

5. Deslandes M, Guillin R, Cardinal E, Hobden R, Bureau NJ. The snapping iliopsoas tendon: new mechanisms using dynamic sonography. AJR Am J Roentgenol 2008;190:576-581.

6. Omar IM, Zoga AC, Kavanagh EC, Koulouris G, Bergin D, Gopez AG, et al. Athletic pubalgia and "sports hernia": optimal MR imaging technique and findings. Radiographics 2008;28:1415-1438.

7. Morley N, Grant T, Blount K, Omar I. Sonographic evaluation of athletic pubalgia. Skeletal Radiol 2016;45:689-699.

8. Zoga AC, Kavanagh EC, Omar IM, Morrison WB, Koulouris G, Lopez $H$, et al. Athletic pubalgia and the "sports hernia": MR imaging findings. Radiology 2008;247:797-807.

9. Hopkins JN, Brown W, Lee CA. Sports hernia: definition, evaluation, and treatment. JBJS Rev 2017;5:e6.

10. Hasselman CT, Best TM, Hughes C 4th, Martinez S, Garrett WE $J$ r. An explanation for various rectus femoris strain injuries using previously undescribed muscle architecture. Am J Sports Med 1995:23:493-499.

11. Pesquer L, Poussange N, Sonnery-Cottet B, Graveleau N, Meyer $P$, Dallaudiere $B$, et al. Imaging of rectus femoris proximal tendinopathies. Skeletal Radiol 2016;45:889-897.

12. Moraux A, Wawer R, Lefevbre G, Cotten H, Demondion X, Cotten A. An anatomical study of the indirect tendon of the rectus femoris using ultrasonography. Eur Radiol 2015;25:3614-3619.

13. Zini R, Panasci M, Papalia R, Franceschi F, Vasta S, Denaro V. Rectus femoris tendon calcification: arthroscopic excision in 6 top amateur athletes. Orthop J Sports Med 2014;2:2325967114561585.

14. Bianchi S, Martinoli C, Waser NP, Bianchi-Zamorani MP, Federici E, Fasel J. Central aponeurosis tears of the rectus femoris: sonographic findings. Skeletal Radiol 2002;31:581-586.

15. Hughes C 4th, Hasselman CT, Best TM, Martinez S, Garrett WE Jr. Incomplete, intrasubstance strain injuries of the rectus femoris muscle. Am J Sports Med 1995;23:500-506.

16. Salati U, Doody O, Munk PL, Torreggiani W. Evaluation of knee pain in athletes: a radiologist's perspective. Can Assoc Radiol J 2017;68:27-40.

17. Pfirrmann CW, Jost B, Pirkl C, Aitzetmuller G, Lajtai G. Quadriceps tendinosis and patellar tendinosis in professional beach volleyball players: sonographic findings in correlation with clinical symptoms. Eur Radiol 2008;18:1703-1709.

18. Kary JM. Diagnosis and management of quadriceps strains and contusions. Curr Rev Musculoskelet Med 2010;3:26-31.

19. La S, Fessell DP, Femino JE, Jacobson JA, Jamadar D, Hayes C. Sonography of partial-thickness quadriceps tendon tears with surgical correlation. J Ultrasound Med 2003;22:1323-1329.

20. Foley R, Fessell D, Yablon C, Nadig J, Brandon C, Jacobson J. Sonography of traumatic quadriceps tendon tears with surgical correlation. J Ultrasound Med 2015;34:805-810.

21. Wangwinyuvirat $M$, Dirim B, Pastore $D$, Pretterklieber $M$, Frank A, Haghighi $P$, et al. Prepatellar quadriceps continuation: MRI of cadavers with gross anatomic and histologic correlation. AJR Am J Roentgenol 2009;192:W111-W116.

22. Gemignani M, Busoni F, Tonerini M, Scaglione M. The patellar tendinopathy in athletes: a sonographic grading correlated to prognosis and therapy. Emerg Radiol 2008;15:399-404.

23. Khan KM, Bonar F, Desmond PM, Cook JL, Young DA, Visentini PJ, et al. Patellar tendinosis (jumper's knee): findings at histopathologic examination, US, and MR imaging. Victorian Institute of Sport Tendon Study Group. Radiology 1996;200:821-827.

24. Kwak HS, Han YM, Lee SY, Kim KN, Chung GH. Diagnosis and follow-up US evaluation of ruptures of the medial head of the gastrocnemius ("tennis leg"). Korean J Radiol 2006;7:193-198.

25. Delgado GJ, Chung CB, Lektrakul N, Azocar P, Botte MJ, Coria D, et al. Tennis leg: clinical US study of 141 patients and anatomic investigation of four cadavers with MR imaging and US. Radiology 2002;224:112-119.

26. Kwak HS, Lee KB, Han YM. Ruptures of the medial head of the gastrocnemius ("tennis leg"): clinical outcome and compression effect. Clin Imaging 2006;30:48-53.

27. Bianchi S, Sailly M, Molini L. Isolated tear of the plantaris tendon: ultrasound and MRI appearance. Skeletal Radiol 2011;40:891-895.

28. Helms CA, Fritz RC, Garvin GJ. Plantaris muscle injury: evaluation with MR imaging. Radiology 1995;195:201-203.

29. Park JW, Lee SJ, Choo HJ, Kim SK, Gwak HC, Lee SM. Ultrasonography of the ankle joint. Ultrasonography 2017;36:321. 335.

30. Calleja M, Connell DA. The Achilles tendon. Semin Musculoskelet Radiol 2010;14:307-322.

31. Edama M, Kubo M, Onishi H, Takabayashi T, Inai T, Yokoyama E, et al. The twisted structure of the human Achilles tendon. Scand J Med Sci Sports 2015;25:e497-e503.

32. Harris CA, Peduto AJ. Achilles tendon imaging. Australas Radiol 2006:50:513-525.

33. Hartgerink P, Fessell DP, Jacobson JA, van Holsbeeck MT. Full- versus partial-thickness Achilles tendon tears: sonographic accuracy and characterization in 26 cases with surgical correlation. Radiology 2001;220:406-412.

34. Ohberg L, Lorentzon R, Alfredson H. Good clinical results but persisting side-to-side differences in calf muscle strength after surgical treatment of chronic Achilles tendinosis: a 5-year follow- 
up. Scand J Med Sci Sports 2001;11:207-212.

35. Astrom M, Gentz CF, Nilsson P, Rausing A, Sjoberg S, Westlin $\mathrm{N}$. Imaging in chronic achilles tendinopathy: a comparison of ultrasonography, magnetic resonance imaging and surgical findings in 27 histologically verified cases. Skeletal Radiol 1996;25:615620.

36. Villarreal AD, Andersen CR, Panchbhavi VK. A survey on management of chronic achilles tendon ruptures. Am J Orthop (Belle Mead NJ) 2012;41:126-131.

37. Lee SJ, Jacobson JA, Kim SM, Fessell D, Jiang Y, Dong Q, et al. Ultrasound and MRI of the peroneal tendons and associated pathology. Skeletal Radiol 2013;42:1191-1200.

38. Bianchi S, Delmi M, Molini L. Ultrasound of peroneal tendons. Semin Musculoskelet Radiol 2010;14:292-306.

39. Neustadter J, Raikin SM, Nazarian LN. Dynamic sonographic evaluation of peroneal tendon subluxation. AJR Am J Roentgenol 2004; 183:985-988.

40. Raikin SM, Elias I, Nazarian LN. Intrasheath subluxation of the peroneal tendons. J Bone Joint Surg Am 2008;90:992-999.

41. Campbell DG, Menz A, Isaacs J. Dynamic ankle ultrasonography: a new imaging technique for acute ankle ligament injuries. Am J Sports Med 1994;22:855-858.

42. Sconfienza LM, Orlandi D, Lacelli F, Serafini G, Silvestri E. Dynamic high-resolution US of ankle and midfoot ligaments: normal anatomic structure and imaging technique. Radiographics 2015:35:164-178.

43. Hua $Y$, Yang $Y$, Chen $S$, Cai Y. Ultrasound examination for the diagnosis of chronic anterior talofibular ligament injury. Acta Radiol 2012;53:1142-1145.

44. Durkee NJ, Jacobson JA, Jamadar DA, Femino JE, Karunakar MA, Hayes CW. Sonographic evaluation of lower extremity interosseous membrane injuries: retrospective review in 3 patients. J Ultrasound Med 2003;22:1369-1375.

45. Mei-Dan O, Kots E, Barchilon V, Massarwe S, Nyska M, Mann G. A dynamic ultrasound examination for the diagnosis of ankle syndesmotic injury in professional athletes: a preliminary study. Am J Sports Med 2009;37:1009-1016.

46. Miller BS, Downie BK, Johnson PD, Schmidt PW, Nordwall SJ, Kijek $\mathrm{TG}$, et al. Time to return to play after high ankle sprains in collegiate football players: a prediction model. Sports Health 2012;4:504509.

47. Kramer DE, Pace JL, Jarrett DY, Zurakowski D, Kocher MS, Micheli LJ. Diagnosis and management of symptomatic muscle herniation of the extremities: a retrospective review. Am J Sports Med 2013;41:2174-2180.

48. Zhou X, Zhan W, Chen W, Wu D, Wei K, Wu C, et al. The value of ultrasound in the preoperative diagnosis of muscle herniation: a comparison with magnetic resonance imaging. Eur J Radiol 2017;94:191-194.

49. Beggs I. Sonography of muscle hernias. AJR Am J Roentgenol 2003; 180:395-399.

50. Cardinal E, Chhem RK, Beauregard CG, Aubin B, Pelletier M. Plantar fasciitis: sonographic evaluation. Radiology 1996;201:257-259.

51. Elias DA, Carne A, Bethapudi S, Engebretsen L, Budgett R, O'Connor P. Imaging of plantar fascia and Achilles injuries undertaken at the London 2012 Olympics. Skeletal Radiol 2013;42:1645-1655.

52. Fleischer AE, Albright RH, Crews RT, Kelil T, Wrobel JS. Prognostic value of diagnostic sonography in patients with plantar fasciitis. J Ultrasound Med 2015;34:1729-1735.

53. Ashman CJ, Klecker RJ, Yu JS. Forefoot pain involving the metatarsal region: differential diagnosis with $M R$ imaging. Radiographics 2001;21:1425-1440.

54. Linklater JM. Imaging of sports injuries in the foot. AJR Am J Roentgenol 2012;199:500-508.

55. Gregg J, Silberstein M, Schneider T, Marks P. Sonographic and MRI evaluation of the plantar plate: a prospective study. Eur Radiol 2006; 16:2661-2669.

56. Fernandes EA, Mann TS, Puchnick A, Tertulino FF, Cannato CT, Nery $C$, et al. Can ultrasound of plantar plate have normal appearance with a positive drawer test? Eur J Radiol 2015;84:443-449.

57. Pathria MN, Chung CB, Resnick DL. Acute and stress-related injuries of bone and cartilage: pertinent anatomy, basic biomechanics, and imaging perspective. Radiology 2016;280:21-38.

58. Drakonaki EE, Garbi A. Metatarsal stress fracture diagnosed with high-resolution sonography. J Ultrasound Med 2010;29:473-476.

59. Wang CL, Shieh JY, Wang TG, Hsieh FJ. Sonographic detection of occult fractures in the foot and ankle. J Clin Ultrasound 1999;27:421-425. 